\title{
Dinámica ecosistémica y calidad de agua: estrategias de monitoreo para la gestión de servicios asociados a Laguna del Sauce (Maldonado, Uruguay)
}

\section{Ecosystem dynamics and water quality: monitoring strategies for the management of Sauce Lake (Maldonado, Uruguay) services}

Crisci, Carolina (1); Goyenola, Guillermo (2); Terra, Rafael (3); Lagomarsino, Juan José (4); Pacheco, Juan Pablo (2); Díaz, Ismael (5); González-Madina, Lucía (2); Levrini, Paula (2); Méndez, Gustavo (4); Bidegain, Mario (6);

Ghattas, Badih (7); Mazzeo, Néstor (2).

(1) Polo de Desarrollo Universitario Modelización y Análisis de Recursos Naturales, Centro Universitario Regional

del Este (CURE), Universidad de la República (UdelaR), Rocha, Uruguay.

(2) Departamento de Ecología Teórica y Aplicada, Centro Universitario Regional del Este (CURE), UdelaR, Maldonado, Uruguay.

(3) Instituto de Mecánica de los Fluidos e Ingeniería Ambiental, Facultad de Ingeniería, UdelaR, Montevideo, Uruguay.

(4) Obras Sanitarias del Estado (OSE), Unidad de Gestión Desconcentrada, Uruguay.

(5) Laboratorio de Desarrollo Sustentable y Gestión Ambiental del Territorio. Instituto de Ecología

y Ciencias Ambientales. Facultad de Ciencias. UdelaR, Montevideo, Uruguay.

(6) Instituto Uruguayo de Meteorología, Uruguay.

(7) Institut de Mathématiques de Marseille, UMR 7373, CNRS, Francia.

Contacto: carocrisci@cure.edu.uy

RECIBIDO: 5/4/2017 -APROBADO: 14/6/2017

\begin{abstract}
Resumen
La eutrofización constituye uno de los principales problemas de los ecosistemas acuáticos continentales. Una de las consecuencias más conocidas de este proceso son las floraciones de algas y cianobacterias que interfieren en el adecuado funcionamiento de los ecosistemas acuáticos y en los servicios que estos brindan. En consecuencia, resulta relevante el desarrollo de estrategias de monitoreo que permitan conocer el funcionamiento de los sistemas para anticipar la evolución temporal del fitoplancton y sus efectos en la calidad del agua, de modo de aportar información trascendente en los procesos de toma decisión en diferentes escalas espacio-temporales. Este trabajo pretende contribuir a ese desarrollo, sistematizando la información hoy disponible en lo referente a monitoreo y análisis de los procesos subyacentes a la dinámica del fitoplancton en Laguna del Sauce (Maldonado, Uruguay). Se identifican los principales avances, dificultades y desafíos, enfatizando la necesidad de la integración de la información y de los procesos de análisis generados por diversas instituciones, así como el planteo de estrategias que promuevan la permanencia en el tiempo de estas prácticas.

Palabras clave: Eutrofización, dinámica del fitoplancton, floraciones de cianobacterias, variabilidad espacio-temporal, análisis y sistematización de la información, procesos de toma de decisión.
\end{abstract}

\section{Abstract}

Eutrophication represents one of the main problems concerning continental aquatic ecosystems. An usual and undesired consequence of eutrophication is the occurrence of algal and cyanobacterial blooms, which hinders the proper functioning of these ecosystems and affects the services that they provide. Therefore, it is important to gain knowledge on the system's functioning with the purpose to anticipate the temporal evolution of phytoplankton dynamics and their effects in the water quality. A key requirement is to generate monitoring strategies, which, in turn, may provide relevant information in decision-making processes regarding a variety of problems at different spatial-temporal scales. In this regard, this study intends to contribute to the systematization of available information that results from the monitoring and analyses of the processes underlying phytoplankton dynamics in Sauce Lake (Maldonado, Uruguay). Relevant progress, problems and challenges are identified and discussed. Finally, we highlight the importance of the integration of the information and the analyses generated by different institutions as well as the need to maintain these practices over time.

Keywords: Eutrophication, phytoplankton dynamics, cyanobacterial blooms, spatio-temporal variability, data analysis and systematization, decision-making processes. 


\section{Introducción}

El aporte externo de nutrientes configura una de las principales alteraciones de la calidad del agua de los ecosistemas acuáticos continentales (Smith y Schindler, 2009; Carpenter, et al., 2011). Las consecuencias de este proceso, denominado eutrofización, son múltiples y pueden afectar diversas actividades humanas como la recreación, la pesca, la navegación y el suministro de agua potable (Carpenter, et al., 2011). La eutrofización se caracteriza por una dinámica temporal de gran complejidad, con cambios de régimen bruscos, retardo en la observación de respuestas e histéresis (Scheffer, et al., 1993; Scheffer y van Nes, 2007), lo que establece múltiples desafíos a la hora del análisis de la información y de la toma de decisión relacionados con la gestión y uso de los recursos acuáticos y cuencas asociadas.

Una de las posibles consecuencias de la eutrofización es la floración o crecimiento no controlado de productores primarios, esto es, macrófitas y fitoplancton (constituido por microalgas y cianobacterias) (Heisler, et al, 2008). Las interferencias más significativas de las floraciones de microalgas y cianobacterias en los procesos de potabilización derivan de la generación de sustancias que alteran el olor y sabor del agua y, en el caso particular de las cianobacterias, de la producción de metabolitos tóxicos (cianotoxinas) (Carmichael, 2001).

La humanidad ha alterado los ciclos de nutrientes (Gruber y Galloway, 2008; Elser y Bennett, 2011), aumentando la magnitud y frecuencia de este tipo de fenómenos.

Como consecuencia, los responsables de usinas de potabilización se ven enfrentados a desafíos crecientes. La capacidad de planificación y anticipación depende de la comprensión de los procesos ecosistémicos de los sistemas acuáticos fuente. En este nuevo contexto resulta indispensable sustentar el proceso de toma decisiones en la generación de conocimiento basado en sólidos programas de monitoreo que permitan conocer el estado del sistema y su dinámica a diferentes escalas espacio-temporales.

Este trabajo pretende contribuir a ese desarrollo, tomando como caso de estudio una de las principales fuentes de agua del país (Laguna del Sauce, Maldonado, Uruguay), mediante:

a) la sistematización de la información hoy disponible en lo referente al monitoreo y al análisis de variables de calidad de agua y en particular a los procesos subyacentes a la dinámica del fitoplancton;

b) la evaluación de los distintos sistemas de monitoreo existentes a la fecha en función de su capacidad de responder a objetivos concretos;

c) la identificación de los principales avances, dificultades y desafíos del actual sistema de seguimiento.

\section{Materiales y Métodos}

\section{Sistema de estudio}

La Laguna del Sauce ( $34^{\circ} 43^{\prime}$ S, $55^{\circ} 13^{\prime} \mathrm{W}$, Maldonado, Uruguay, Figuras la y 1b) está conformada por un sistema de tres lagunas someras (profundidad media 2,5 metros) conectadas: El Sauce (4045 ha), De los Cisnes (205 ha) y El Potrero (411 ha) (Figura 1b). El sistema Laguna del Sauce ha sido clasificado como eutrófico; el fósforo total ha alcanzado niveles mayores

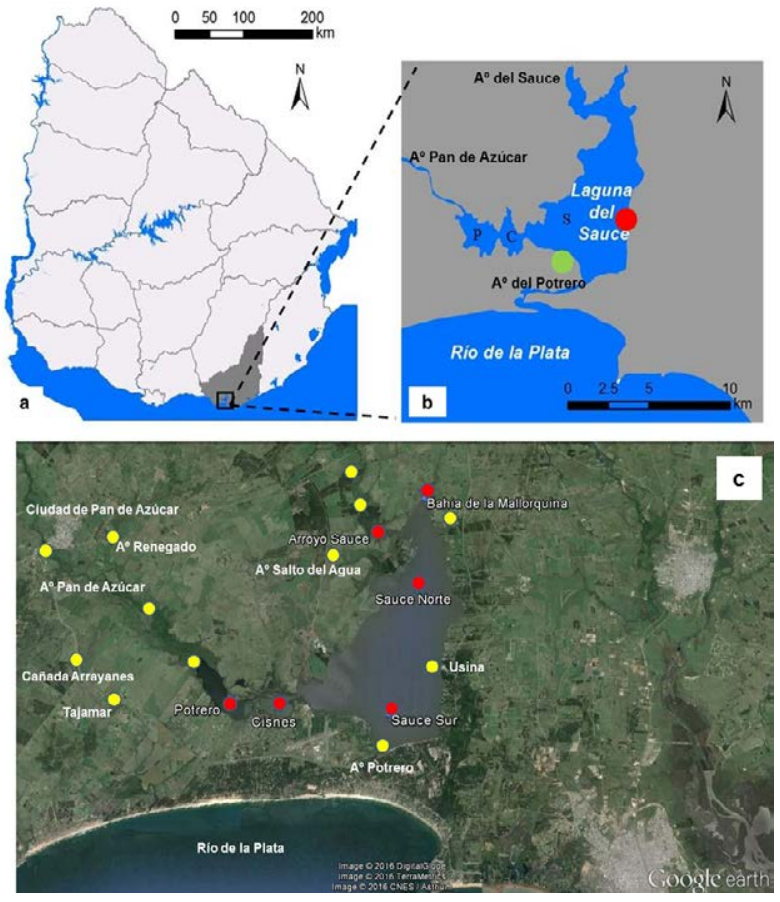

Figura 1. Sistema Laguna del Sauce. a) El Sistema Laguna del Sauce se ubica en el Departamento de Maldonado (sureste de Uruguay). b) El sistema se conforma de tres lagos someros conectados: Laguna del Sauce (S), Laguna De los Cisnes (C) y Laguna del Potrero (P). El círculo rojo indica la ubicación de la usina de OSE-UGD (Unidad de Gestión Desconcentrada) y el verde muestra la localización de la estación meteorológica del Instituto Uruguayo de Meteorología. Se muestran también los arroyos del Sauce y Pan de Azúcar (principales tributarios) y el arroyo del Potrero (conexión natural con el Río de la Plata). c) Mapa del Sistema Laguna del Sauce donde se indican los puntos de muestreo de las campañas semanales (puntos rojos) y los puntos que se adicionan en las campañas mensuales (puntos amarillos; ver subsección Muestreos semanales y mensuales de información limnológica en el cuerpo de agua y red de tributarios de la sección Materiales y Métodos).

a $80 \mathrm{ug} / \mathrm{L}$ en varias ocasiones (y valores mayores a $100 \mathrm{ug} / \mathrm{L}$ en períodos de bajo nivel del agua; Inda y Steffen, 2010). El nitrógeno total presenta una importante variabilidad temporal, con valores que van de 200 a $1000 \mathrm{ug} / \mathrm{L}$ (Inda y Steffen, 2010). El sistema presenta un complejo patrón espacial de respuestas a la eutrofización. En la Laguna del Potrero se observa un crecimiento no controlado de vegetación acuática, mientras que en el resto del sistema (Laguna del Sauce y De los Cisnes) se registran, fundamentalmente en épocas estivales, floraciones de cianobacterias, concretamente de diversas especies fijadoras de nitrógeno (e.g. Aphanizomenon spp., Dolichospermum spp.) y productoras potenciales de cianotoxinas, principalmente microcistina (hepatotoxina) y saxitoxina (neurotoxina). Cabe destacar que durante otoño de 2015 y diciembre del mismo año las floraciones de cianobacterias han provocado diversas crisis en el suministro de agua potable, con múltiples repercusiones a nivel de los medios de difusión (Muñoz, 2015; El Observador, 2015).

La Laguna del Sauce constituye la segunda fuente de agua potable del país en términos de personas abastecidas (de 140.000 
personas en temporada baja a 400.000 personas en temporada estival). La Unidad de Gestión Desconcentrada de OSE (OSEUGD), ubicada en el margen este de la laguna (Figura 1b), extrae agua desde fines de la década de 1970 (Inda y Steffen, 2010).

\section{Estrategias de monitoreo disponibles en Laguna del Sauce}

Para la generación de un sistema de seguimiento integral de calidad de agua es necesario considerar la gestión de toda la cuenca en el mediano y largo plazo a efectos de asegurar la sustentabilidad del sistema y los servicios que presta. Esta información debe complementarse con una adecuada comprensión de la dinámica general del sistema, de la variabilidad climática y de eventos extremos. De esta forma es posible definir indicadores apropiados y sistemas de monitoreo acordes a los procesos de gestión de largo aliento. Dentro del marco de la gestión integral conviven otros procesos de toma de decisión más rápidos -y generalmente más localizados- que requieren de herramientas técnicas e institucionales aptas para dar respuestas oportunas. En particular, es decisivo construir capacidades para anticipar y detectar cambios abruptos de calidad de agua que permitan aplicar medidas preventivas o de mitigación. En sistemas eutróficos como Laguna del Sauce, estos eventos se asocian generalmente a floraciones fitoplanctónicas que ocasionan múltiples problemas de sabor, olor y toxicidad. En este marco, se requiere contar con indicadores simples y rápidos de obtener y modelos predictivos de floraciones aplicables en tiempo real. En todos los casos se necesita el desarrollo de sistemas de monitoreo que permitan conocer el estado del sistema y anticipar, en combinación con los modelos, su evolución futura. El siguiente nivel de decisión ocurre cuando se verifica la presencia de floraciones algales, lo cual puede disparar otra serie de medidas, como los múltiples ajustes en los procesos de potabilización que pueden incluir modificaciones de los procesos rutinarios, entre otros, los cambios en la dosificación de cloro, el pretratamiento con carbono en polvo, dióxido de cloro o permanganato.

En esta sección se describen los sistemas de monitoreo disponibles que atienden procesos a distintas escalas espacio-temporales y que, por tanto, pretenden responder a objetivos diversos que en su conjunto intentan conformar un sistema integral de seguimiento de calidad de agua. La Tabla 1 presenta una síntesis de todos los sistemas disponibles a la fecha descriptos en esta sección y sus principales características.

Registro de información

meteorológica e hidrológica

Existe información meteorológica de la estación meteorológica de Laguna del Sauce (WMO No86586; Figura 1b) perteneciente al Instituto Uruguayo de Meteorología (INUMET). Desde el 2002 a la fecha se dispone de información digitalizada a paso diario de precipitación acumulada, temperatura mínima, máxima y media, y velocidad media del viento (Tabla 1). Las temperaturas y la velocidad del viento a paso diario se calculan a partir de datos tri-horarios obtenidos de mensajes SYNOP (surface synoptic observations). Para el caso del viento, la información tri-horaria incluye, además de velocidad, dirección.

Estas medidas se realizan en la estación meteorológica a 1,5 metros de altura (Tabla 1). Por otra parte, se dispone de datos diarios de nivel de agua en la laguna, relevados desde 1970, aportados por la Dirección Nacional de Agua (DINAGUA; Tabla 1).

\section{Objetivos del monitoreo}

La obtención de información meteorológica e hidrológica (observada y de pronóstico) en tiempo real por parte de los funcionarios de OSE-UGD tiene como objetivo alertar sobre posibles respuestas del sistema frente a ocurrencia de determinados eventos, de modo de brindar información sobre potenciales cambios en variables clave de calidad del agua a operarios de la Usina de OSE-UGD. Estas respuestas a determinados eventos meteorológicos e hidrológicos han sido constatadas mediante estudios recientes que emplean modelos predictivos (Crisci, et al., en prensa).

Por otra parte, el registro continuo de este tipo de información también tiene como objetivo el aporte de nuevos datos para la mejora de los modelos predictivos mencionados (sección Resultados, subsección Registro de información hidrológica y meteorológica; Figura 2).

\section{Monitoreo de información limnológica \\ en la planta de OSE-UGD}

A partir del monitoreo realizado por OSE-UGD para evaluar la calidad del agua utilizada por la planta potabilizadora se genera información diaria de numerosos parámetros como temperatura, turbidez, $\mathrm{pH}$, conductividad, alcalinidad, color del agua, nitritos, nitratos, amonio y biomasa algal (clorofila- $a$ ). Esta información se encuentra disponible desde el año 2002.

Desde el año 2004 se registra con frecuencia diaria/semanal/quincenal la abundancia de fitoplancton (org/ml) de todas las especies identificadas (monitoreo hidrobiológico). La aparición de cianobacterias determina la implantación de un monitoreo hidrobiológico diario de agua bruta (i.e. agua de la laguna que no ha recibido ningún tratamiento) y del resto del proceso de potabilización. Este monitoreo incluye la observación hidrobiológica durante todo el desarrollo del evento y, desde fines de 2014, la cuantificación de toxinas mediante el método de lectura en placas con kits específicos para microcistinas, anatoxina, saxitoxina y cilindrospermopsina. Por último, desde el año 2013 se realiza un monitoreo quincenal de fósforo total (PT) y mensual de nitrógeno total (NT).

\section{Objetivos del monitoreo}

El objetivo principal del monitoreo continuo realizado por OSE-UGD es la evaluación de calidad del agua en tiempo real, lo que deriva en el ajuste del proceso de potabilización con el fin de prevenir o mitigar problemas en la calidad de agua para consumo humano.

Además de la operativa de la planta, al igual que para el registro de información meteorológica e hidrológica, la información obtenida por OSE-UGD es uno de los insumos principales para la comprensión de procesos ecosistémicos clave, como la dinámica temporal de variables de calidad de agua y su interrelación con la variabilidad climática, así como para la generación de capacidad predictiva de floraciones fitoplanctónicas mediante la utilización de modelos (Crisci, et al., en prensa).

\section{Sonda de registro continuo}

El 9/03/2016 se instaló una sonda multiparamétrica con transmisión telemétrica de datos en la entrada de agua bruta a la planta potabilizadora. En este monitoreo se utiliza una sonda YSI 6600V2-4 en la que se configuraron sensores de temperatura y conductividad (YSI 6560), pH (YSI 6561), 


\begin{tabular}{|c|c|c|c|c|c|}
\hline $\begin{array}{l}\text { Tipo } \\
\text { de monitoreo }\end{array}$ & Variables registradas & Frecuencia & $\begin{array}{l}\text { Lugar } \\
\text { de registro }\end{array}$ & $\begin{array}{l}\text { Institución } \\
\text { a cargo }\end{array}$ & Objetivo del monitoreo \\
\hline Meteorológico & $\begin{array}{l}\text { Precipitación } \\
\text { acumulada, T media, } \\
\text { máxima y mínima, } \\
\text { velocidad media del } \\
\text { viento/velocidad y } \\
\text { dirección del viento }\end{array}$ & $\begin{array}{l}\text { Diaria/tri- } \\
\text { horaria }\end{array}$ & $\begin{array}{l}\text { Estación } \\
\text { meteorológica } \\
\text { del Laguna del } \\
\text { Sauce (Figura } \\
\text { 1b) }\end{array}$ & INUMET & \multirow{3}{*}{$\begin{array}{l}\text { Seguimiento en tiempo real con } \\
\text { el fin de poder realizar ajustes } \\
\text { en el proceso de potabilización } \\
\text { de modo de prevenir o mitigar } \\
\text { problemas de calidad de agua para } \\
\text { consumo humano / generación de } \\
\text { serie de datos para comprensión } \\
\text { de procesos ecosistémicos y cons- } \\
\text { trucción de modelos predictivos } \\
\text { de calidad de agua }\end{array}$} \\
\hline Hidrológico & Nivel del agua & Diaria & $\begin{array}{l}\text { Laguna } \\
\text { del Sauce } \\
\text { (vertedero) }\end{array}$ & DINAGUA & \\
\hline $\begin{array}{l}\text { Limnológico, } \\
\text { Usina OSE- } \\
\text { UGD }\end{array}$ & $\begin{array}{l}\text { T, turbidez, } \mathrm{pH} \text {, } \\
\text { conductividad, } \\
\text { alcalinidad, color } \\
\text { del agua, nitritos, } \\
\text { nitratos, amonio, clo-al } \\
\text { abundancia especies } \\
\text { de fitoplancton y } \\
\text { cianotoxinas/PT/ NT }\end{array}$ & $\begin{array}{l}\text { Diaria/ } \\
\text { diaria, } \\
\text { semanal o } \\
{\text { quincenal }{ }^{\star} /}^{\text {quincenal/ }} \\
\text { Mensual }\end{array}$ & $\begin{array}{l}\text { Muestras de } \\
\text { agua obtenidas } \\
\text { en la toma de } \\
\text { agua bruta } \\
\text { de la planta } \\
\text { potabilizadora } \\
\text { (100 m offshore } \\
\text { de la Usina de } \\
\text { OSE-UGD) }\end{array}$ & OSE-UGD & \\
\hline $\begin{array}{l}\text { Limnológico, } \\
\text { sonda de } \\
\text { registro } \\
\text { continuo }\end{array}$ & $\begin{array}{l}\text { T, conductividad, } \\
\text { OD, turbidez, clo- } a \\
\text { ficocianina }\end{array}$ & $\begin{array}{l}\text { Cada } 60 \\
\text { segundos }\end{array}$ & $\begin{array}{l}\text { Sonda ubicada } \\
\text { junto a la toma } \\
\text { de la Usina de } \\
\text { OSE-UGD }\end{array}$ & $\begin{array}{l}\text { OSE-UGD, } \\
\text { UdelaR, } \\
\text { DINAMA/ } \\
\text { MVOTMA }\end{array}$ & $\begin{array}{l}\text { Detección automática de } \\
\text { problemas de calidad de agua } \\
\text { para generación de alarmas con } \\
\text { el fin de poder realizar ajustes } \\
\text { en el proceso de potabilización y } \\
\text { prevenir o mitigar problemas de } \\
\text { calidad de agua para consumo } \\
\text { humano }\end{array}$ \\
\hline $\begin{array}{l}\text { Limnológico, } \\
\text { muestreos } \\
\text { semanales y } \\
\text { mensuales en } \\
\text { varios puntos } \\
\text { del sistema }\end{array}$ & $\begin{array}{l}\text { T, conductividad, } \\
\text { STD, pH, potencial } \\
\text { de óxido-reducción, } \\
\text { turbidez, OD, biomasa } \\
\text { de fitoplancton } \\
\text { y cianobacterias, } \\
\text { transparencia del agua, } \\
\text { flujo fotónico, N y P } \\
\text { (formas disueltas y } \\
\text { totales), clo- } a \text {, ST y } \\
\text { contenido de materia } \\
\text { orgánica, composición } \\
\text { y abundancia de fito y } \\
\text { zooplancton }\end{array}$ & $\begin{array}{l}\text { Semanales } \\
\text { y mensuales } \\
\text { (se mide } \\
\text { lo mismo } \\
\text { en ambos } \\
\text { muestreos } \\
\text { pero en los } \\
\text { mensuales } \\
\text { se agregan } \\
\text { puntos de } \\
\text { muestreo) }\end{array}$ & $\begin{array}{l}\text { Varios puntos } \\
\text { del Sistema } \\
\text { Laguna del } \\
\text { Sauce y de sus } \\
\text { tributarios } \\
\text { (Figura 1c) }\end{array}$ & $\begin{array}{l}\text { OSE-UGD, } \\
\text { UdelaR }\end{array}$ & $\begin{array}{l}\text { Comprensión de la variabilidad } \\
\text { espacio-temporal de procesos } \\
\text { ecosistémicos clave/ generación } \\
\text { de insumos para toma de medidas } \\
\text { de prevención o mitigación de } \\
\text { problemas de calidad de agua, } \\
\text { tanto a nivel de la cuenca como } \\
\text { del cuerpo de agua }\end{array}$ \\
\hline
\end{tabular}

Tabla 1. Tipos de monitoreos disponibles en el sistema Laguna del Sauce y principales características. ${ }^{\star}$ Refiere a que el monitoreo de abundancia de especies del fitoplancton y de concentración de cianotoxinas responde a una estrategia adaptativa donde se da un aumento en el registro de la información si se detectan floraciones o especies potencialmente tóxicas. Abreviaciones: T: temperatura, PT Y NT: fósforo y nitrógeno total, clo- $a$ : clorofila $a$, OD: oxígeno disuelto, STD: sólidos totales disueltos, $\mathrm{P}$ : fósforo, $\mathrm{N}$ : nitrógeno, $\mathrm{ST}$ : sólidos totales.

oxígeno disuelto (ROX optic YSI 6150), turbidez (optic YSI 6136), ficocianina (BGA-PC optic YSI 6131) y clorofila- $a$ (optic YSI 6025), propiedad del Centro Universitario Regional del Este de la UdelaR. Cabe considerar que las medidas de clorofila- $a$ y ficocianina por fluorometría in vivo resultan semicuantitativas por razones inherentes al método, y que reflejan cambios proporcionales en el tiempo y no magnitudes absolutas de cada variable.
La sonda fue configurada para realizar lecturas cada 60 segundos y registro de esas mediciones cada 10 minutos, con envío inmediato de datos para integrar la información al proceso de toma de decisiones a tiempo real en la planta de potabilización. La telemetría estuvo a cargo de la empresa Segura Satelital, contratada por OSE-UGD, que utilizó una interfase SDI-12/Modbus Translator (Rogue Ingeneering Inc.) y un datalogger Hermes M102 (Microcom) con capacidad de 
40.000 registros en memoria y envío de datos vía GPRS/SMS. La información histórica y a tiempo real resulta accesible para usuarios registrados vía web, aplicación Windows (Zeus Client) y dispositivos móviles (aplicación Zeus mobile). A la fecha tienen acceso directo a los datos usuarios de OSE, CURE-UdelaR y DINAMA/MVOTMA.

\section{Objetivos del monitoreo}

El objetivo de la instalación de la sonda de registro continuo fue detectar automáticamente cambios abruptos en el comportamiento de las variables que, en función del conocimiento teórico y limnológico específico de la laguna, resultasen indicadores de potenciales problemas de calidad de agua. La detección de cambios abruptos genera alarmas en tiempo real que permiten prevenir o mitigar problemas de calidad del agua en la Usina de OSE-UGD.

De acuerdo al conocimiento previo de la dinámica ecosistémica, se consideró que las variables medidas por la sonda podrían jugar un rol en la alerta temprana respecto al arribo de floraciones fitoplanctónicas a la planta de potabilización (variables relevantes: clorofila- $a$ y ficocianina; turbidez, $\mathrm{pH}$, oxígeno disuelto), aumento del contenido de materia orgánica en descomposición ( $\mathrm{pH}$, oxígeno disuelto), vertidos (conductividad, turbidez, oxígeno disuelto), eventos de resuspensión masiva de sedimentos asociados a tormentas intensas (turbidez), procesos de arrastre de material en suspensión y disuelto luego de eventos intensos de precipitación (turbidez, también clorofila- $a$ y ficocianina), entre otros.

\begin{tabular}{|c|c|c|c|c|c|}
\hline Variable & Alto & Bajo & Histéresis & Persistencia & ¿Qué puede indicar? \\
\hline Temperatura & $30^{\circ} \mathrm{C}$ & $10^{\circ} \mathrm{C}$ & $0,2^{\circ} \mathrm{C}$ & $300 \mathrm{~s}$ & $\begin{array}{c}\text { Temperatura fuera del rango natural } \\
\text { esperable. ¿Falla? }\end{array}$ \\
\hline Conductividad & $300 \mu \mathrm{S} / \mathrm{cm}$ & $70 \mu \mathrm{S} / \mathrm{cm}$ & $1 \mu \mathrm{S} / \mathrm{cm}$ & $300 \mathrm{~s}$ & $\begin{array}{c}\text { Conductividad fuera del rango natural } \\
\text { esperable. ¿Vertido? ¿Falla? }\end{array}$ \\
\hline $\mathrm{pH}$ & 8,8 & 7 & 0,1 & $300 \mathrm{~s}$ & $\begin{array}{c}\text { pH }>8.8 \text { elevada productividad primaria/ } \\
\text { floraciones. } \\
\text { pH }<7 \text { descomposición de materia } \\
\text { orgánica. }\end{array}$ \\
\hline $\begin{array}{l}\text { Variación de } 12 \\
\% \text { de } \mathrm{pH} \text { en } 30 \\
\text { minutos }\end{array}$ & $112 \%$ & $88 \%$ & $5 \%$ & $1 \mathrm{~s}$ & $\begin{array}{l}\text { Se reporta cuando la tasa de cambio } \\
\text { excede una variación de } 12 \% \text { en } \\
\text { ODOsat/hora. Cambios abruptos } \\
\text { derivados del arribo de masas de } \\
\text { agua con floraciones o contaminación } \\
\text { orgánica (u otros). }\end{array}$ \\
\hline $\begin{array}{l}\text { BGA-PC } \\
\text { (ficocianina in } \\
\quad \text { vivo) }\end{array}$ & $\begin{array}{l}5000 \text { y } \\
50000 \text { cel- } \\
\text { PC/mL }\end{array}$ & $\begin{array}{l}-500^{*} \text { cel- } \\
\mathrm{PC} / \mathrm{mL}\end{array}$ & 5 cel-PC/Ml & $300 \mathrm{~s}$ & $\begin{array}{l}\text { Floraciones cianobacterianas, } \\
\text { resuspensión de perifiton. }\end{array}$ \\
\hline Turbidez & 80 & -10 & 0,1 & $300 \mathrm{~s}$ & $\begin{array}{l}\text { Floraciones, resuspensión, arrastre, } \\
\text { vertidos. Los umbrales de las alertas } \\
\text { reportan datos ilógicos (problemas en el } \\
\text { sensor). }\end{array}$ \\
\hline $\begin{array}{l}\text { Clorofila- } a \text { (in } \\
\text { vivo) }\end{array}$ & 20 y $80 \mu \mathrm{g} / \mathrm{L}$ & $-20 \mu \mathrm{g} / \mathrm{L}$ & $1 \mu \mathrm{g} / \mathrm{L}$ & $300 \mathrm{~s}$ & $\begin{array}{l}\text { Floraciones fitoplanctónicas, } \\
\text { resuspensión de perifiton. }\end{array}$ \\
\hline $\begin{array}{l}\text { Oxígeno } \\
\text { disuelto } \\
\text { (porcentaje de } \\
\text { saturación) }\end{array}$ & $120 \%$ & $80 \%$ & $5 \%$ & $1 \mathrm{~s}$ & $\begin{array}{c}\text { Se reporta cuando la tasa de cambio } \\
\text { excede una variación de } 20 \% \text { en } \\
\text { ODOsat/hora. Cambios abruptos } \\
\text { derivados del arribo de masas de agua } \\
\text { con floraciones, contaminación orgánica } \\
\text { (u otros). }\end{array}$ \\
\hline $\begin{array}{l}\text { Variación de } 50 \\
\% \text { de turbidez } \\
\text { en } 10 \text { minutos }\end{array}$ & $150 \%$ & $50 \%$ & $5 \%$ & $1 \mathrm{~s}$ & $\begin{array}{l}\text { Se reporta cuando la tasa de cambio } \\
\text { excede una variación de } 50 \% \text { en } \\
\text { ODOsat/hora. Cambios abruptos } \\
\text { derivados del arribo de masas de } \\
\text { agua con floraciones o contaminación } \\
\text { orgánica (u otros). }\end{array}$ \\
\hline
\end{tabular}

Tabla 2. Consignas para la generación automática de alarmas. Se establecen los límites inferiores (bajo) y superiores (alto) para cada variable considerada, así como la histéresis y persistencia. Recuérdese que las medidas de clorofila- $a$ y ficocianina por fluorometría in vivo resultan semicuantitativas y reflejan cambios proporcionales en el tiempo y no magnitudes absolutas de cada variable, por lo que las alertas generadas en función de estas variables deben ser analizadas en detalle. 
Sobre esta base, se definieron a priori y con participación de técnicos de todas las instituciones involucradas un conjunto de criterios para la generación de alarmas (Tabla 2). Los criterios fueron establecidos definiendo umbrales y tasas de cambio respecto al tiempo. Adicionalmente, se incluyeron alarmas de potencial mal-funcionamiento de los equipos (por ejemplo, valores no esperables de temperatura o turbidez). Para cada variable se establecieron valores de histéresis y persistencia para la generación de las alarmas. La histéresis establece el rango de la variación respecto al valor de consigna para el cual el sistema no genera alarmas. La persistencia, entendida como el tiempo de respuesta o retardo, establece el lapso en segundos por el que debe repetirse una consigna antes de la generación de la alarma. El ajuste de los parámetros de histéresis y persistencia fue realizado empíricamente para eliminar el envío de falsos positivos y el envío repetido de alarmas en lapsos cortos de tiempo (fenómeno frecuentemente nombrado como «traqueteo»). Las alarmas son ruteadas a usuarios predefinidos vía SMS, mail y/o las aplicaciones específicas.

Muestreos semanales y mensuales

de información limnológica en el

cuerpo de agua y red de tributarios

Desde la temporada estival 2015-2016 se realizan muestreos con frecuencia semanal en los siguientes puntos de muestreo: 1) Arroyo Sauce; 2) Bahía de La Mallorquina (extremo Norte de la Laguna del Sauce); 3) Sector Norte; 4) Sector Sur de la Laguna del Sauce; 5) Laguna De los Cisnes, y 6) Laguna del Potrero (Figura 1c).
En estos puntos se registraron in situ, mediante sonda multiparamétrica YSI, temperatura, conductividad, sólidos totales disueltos, $\mathrm{pH}$, potencial de óxido-reducción, turbidez, oxígeno disuelto, biomasa de fitoplancton y cianobacterias. La transparencia del agua fue evaluada mediante disco de Secchi y se registró la densidad de flujo fotónico en superficie, 0,5, 1 y 1,5 metros de profundidad. Adicionalmente, se colectaron muestras de agua para el análisis del contenido de nitrógeno y fósforo, tanto de las formas disueltas como totales (SRP, Murphy y Riley, 1962; nitrato (N-NO-3), Müller y Widemann, 1955; amonio (N-NH+4), Koroleff, 1970; nitrógeno total (TN) y fósforo total (TP), Valderrama, 1981). Además, se colectaron muestras para la determinación de sólidos totales y su contenido de materia orgánica y de clorofila- $a$ mediante extracción fría con etanol (Nusch, 1980). Finalmente, se obtuvieron muestras integradas de la columna de agua y por triplicado para el análisis de la composición y abundancia del fito y zooplancton.

Con una marcha mensual se realizó un muestreo sobre las estaciones de muestreo descriptas, relevando la misma información indicada, pero se adicionaron diversos puntos de muestreo en el sistema de tributarios (Figura 1c).

\section{Objetivos del monitoreo}

Con los muestreos semanales y mensuales se pretende evaluar aspectos fundamentales del sistema, como su estado trófico, principales respuestas al proceso de eutrofización, localización espacial de las diferentes respuestas, principales características de la red de tributarios y concentraciones de

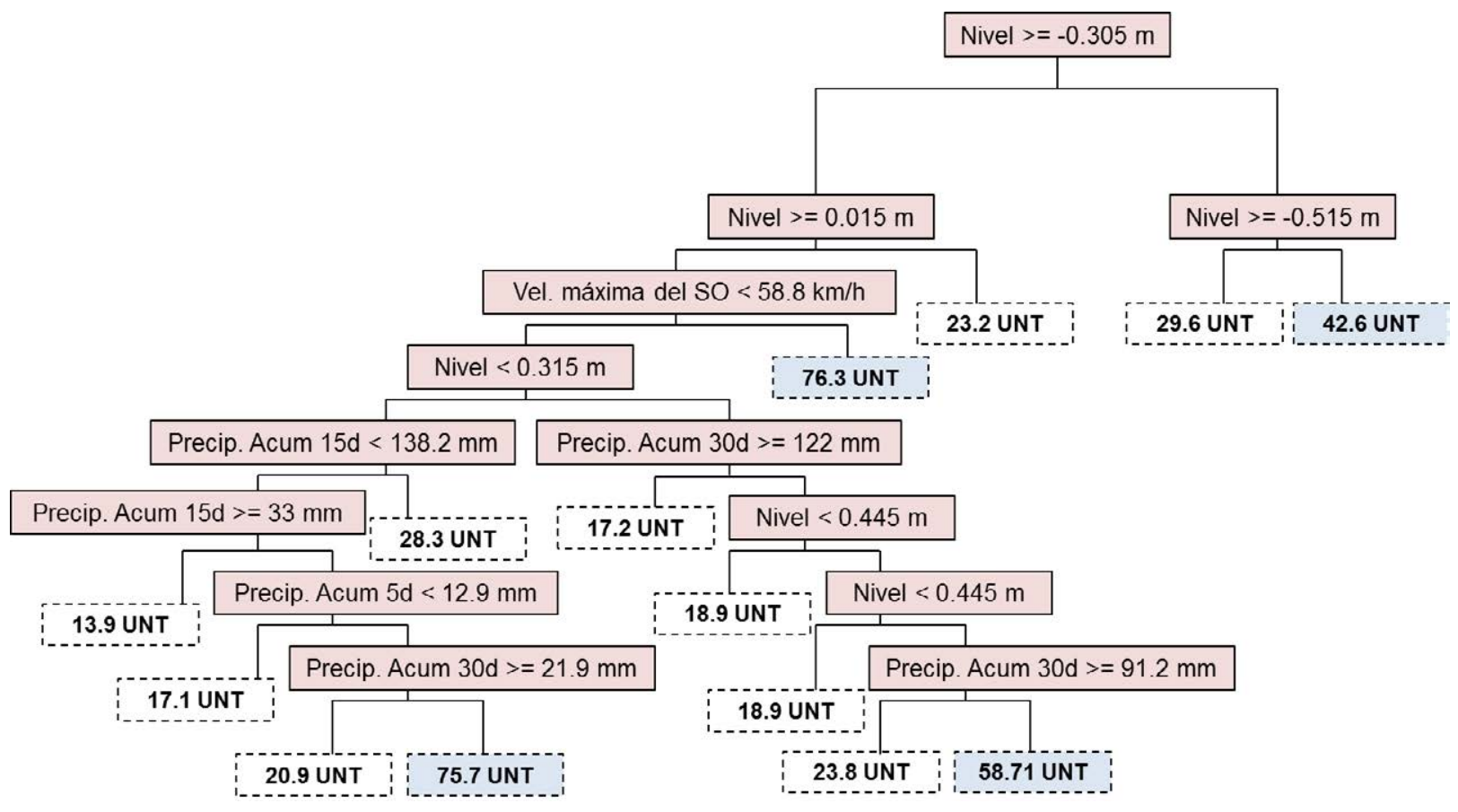

Figura 2. Árbol de regresión para predicción de eventos extremos de turbidez (rectángulos celestes) e identificación de condiciones meteorológicas e hidrológicas que los desencadenan (figura adaptada de Crisci, et al., en prensa). 
nutrientes aportadas, entre otros. La finalidad es generar insumos para toma de medidas de prevención o mitigación de problemas de calidad de agua tanto a nivel de la cuenca como del cuerpo de agua.

\section{Resultados}

\section{Registro de información hidrológica y meteorológica}

La información meteorológica e hidrológica disponible resultó de gran utilidad para la comprensión de la dinámica temporal de variables clave de calidad de agua como la turbidez. En este sentido, modelos estadísticos (en particular Arboles de Regresión; Breiman, et al., 1984) permitieron generar predicciones sobre condiciones extremas de turbidez que dan comienzo a períodos prolongados de elevados valores de esta variable (períodos en los que el crecimiento del fitoplancton se ve limitado; Crisci, et al., en prensa; Figura 2, Figura 3a), a partir de información de variables meteorológicas e hidrológicas (Figura 2).

Por otro lado, la utilización de información meteorológica e hidrológica en tiempo real para anticipar condiciones en la calidad de agua en base a conocimientos previos (resultados anteriores de los análisis de dinámica temporal de las variables y de modelos estadísticos), es limitada, ya que no existe una transferencia automática de información observada ni de pronóstico desde INUMET a OSE-UGD. En consecuencia, la generación de predicciones de modelos en tiempo real no está aún implementada.

A continuación, se presentan resultados de la modelización estadística mediante la utilización de Árboles de regresión (Figura 2).

Algunos ejemplos de respuestas del sistema a eventos meteorológicos e hidrológicos son:

- Vientos intensos (velocidad mayor a $\sim 40 \mathrm{~km} / \mathrm{h}$ ) de la dirección suroeste (SO) pueden generar eventos importantes de resuspensión y modificar por completo la dinámica del sistema, aumentando de forma drástica la turbidez inorgánica y limitando la biomasa fitoplactónica por períodos prolongados (Crisci, et al., en prensa).

- Eventos extremos de precipitación o elevados valores acumulados en el lapso de una a cuatro semanas, que ocurren solos o en combinación con vientos del SO moderados a fuertes, también pueden generar aumentos de turbidez inorgánica y cambios en la dinámica de la laguna a más largo plazo (Crisci, et al., en prensa).

- Bajos niveles agua en combinación con vientos moderados a fuertes constituyen otra combinación de condiciones que pueden dar lugar a aumentos drásticos de turbidez por períodos prolongados (Crisci, et al., en prensa).

\section{Monitoreo de información limnológica en la planta de OSE-UGD}

El registro de esta información viene resultando de gran utilidad para atender el objetivo principal de este tipo de monitoreo que constituye la evaluación de calidad del agua en tiempo real para prevenir o mitigar problemas en la calidad de agua.

En concreto, mediante el registro continuo de biomasa (clorofila-a), abundancia de especies de fitoplancton y toxicidad de especies potencialmente tóxicas, este monitoreo ha permitido la detección temprana de floraciones fitoplanctónicas y su toxicidad, lo que ha posibilitado activar y optimizar la batería mitigatoria disponible para estos episodios. Otros parámetros como el $\mathrm{pH}$ y niveles de materia orgánica han sido de gran importancia para detectar y atacar problemas de carácter físicoquímico como el sabor y el olor. Muchas veces estos problemas ocurren durante o inmediatamente después de eventos de floraciones y su remoción constituye hoy un desafío a nivel mundial, en particular cuando hay presencia de metabolitos de olor y sabor (asociados a geosmina, 2-metilisoborenol).

Además de estos aspectos, la información obtenida por OSE-UGD es uno de los insumos principales para la comprensión de procesos ecosistémicos, como la dinámica temporal de variables de calidad de agua y su interrelación con la variabilidad climática, así como para la generación de capacidad predictiva de floraciones fitoplanctónicas.

En este sentido, estudios recientes (Crisci, et al., en prensa) han permitido conocer el papel de importantes forzantes externas en la dinámica fitoplanctónica. En síntesis, la dinámica fitoplanctónica es gobernada por fluctuaciones particulares de la turbidez de naturaleza inorgánica y otras forzantes físicas asociadas al nivel del agua y al tiempo de residencia. La variación temporal de la turbidez depende de la dinámica de eventos extremos de viento (de dirección SO) y/o precipitación, o eventos moderados de viento cuando el nivel de agua es bajo. Al mismo tiempo, los niveles de agua se asocian a períodos de sequía donde aumentan los tiempos de residencia que favorecen a las especies con tasas de crecimiento más lento, fundamentalmente cianobacterias. Los procesos físicos explican un importante porcentaje de las grandes variaciones temporales de la comunidad fitoplanctónica en este sistema. Las investigaciones de Crisci et al. (en prensa) han sido posibles gracias al registro diario de información limnológica en combinación con información meteorológica e hidrológica, así como al análisis de la comunidad fitoplanctónica en un régimen adaptativo de muestreo. En este caso, fue necesaria la combinación de diferentes aproximaciones matemáticas (análisis de series temporales; técnicas de Machine Learning como Árboles de Regresión y Clasificación -Breiman, et al., 1984; Breiman, 2001-; y ecuaciones físicas de resuspensión del sedimento -Clifton y Dingler, 1984-). En particular, los modelos estadísticos (técnicas de Machine Learning) mostraron un desempeño aceptable en la predicción de Grupos Funcionales Basados en Morfología (Kruk, et al., 2010) de fitoplancton. La incorporación de nueva información generada en el futuro podrá mejorar la capacidad predictiva de los modelos.

Al momento, este tipo de información es accesoria a la información obtenida en tiempo real y puede contribuir aún de forma limitada a la toma de decisiones. Aunque puede aportar, por ejemplo, en la redefinición de estrategias de monitoreo de ciertos parámetros y dar información complementaria sobre respuestas del sistema a ciertas forzantes.

La Figura 3 presenta resultados del análisis de información diaria recabada en la planta de OSE-UGD durante 11 años, considerando diferentes aproximaciones. Como se mencionó en la subsección anterior, estos análisis permitieron conocer 

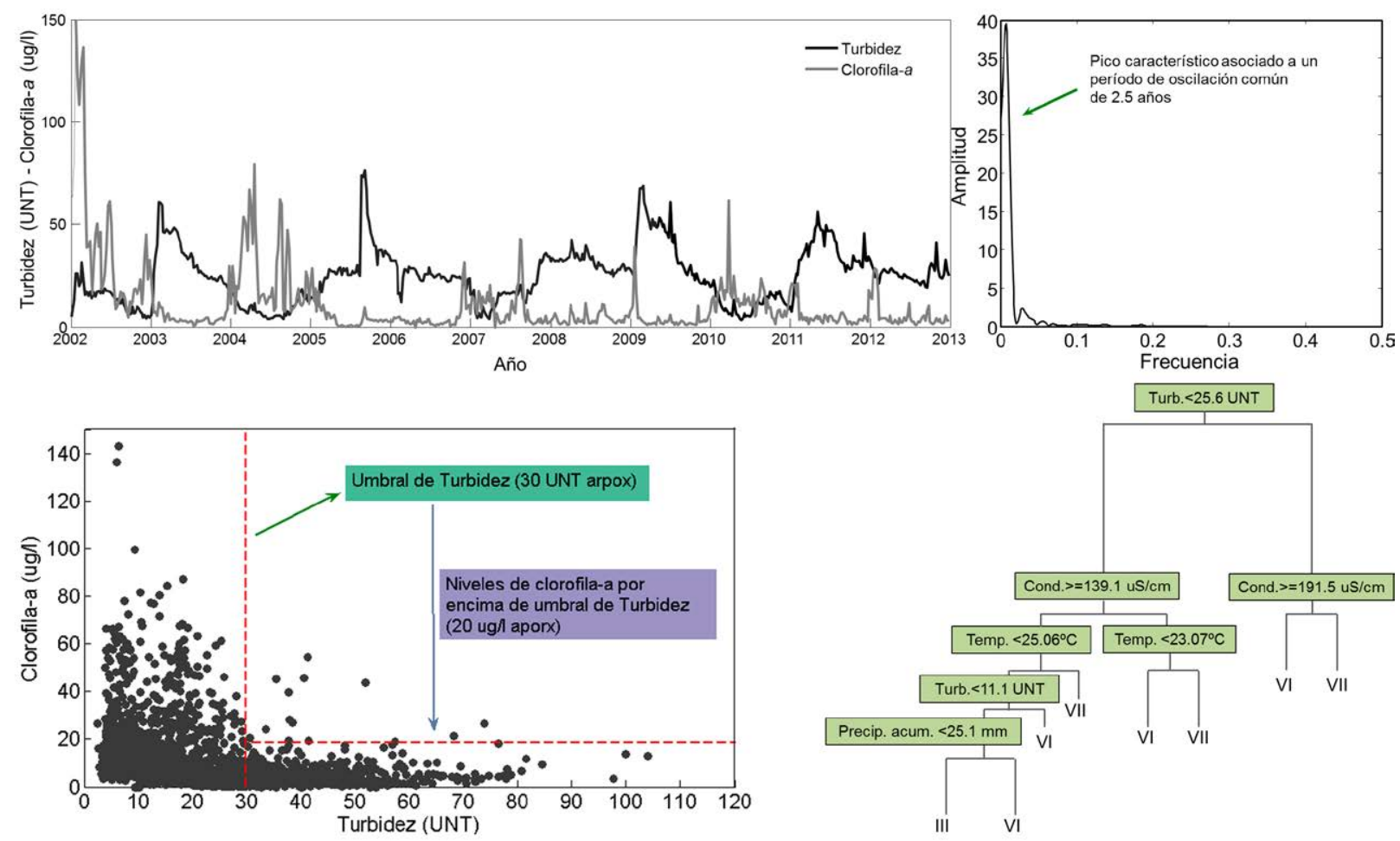

Figura 3. Resultados de análisis a partir de información diaria registrada en la planta de OSE-UGD. a) Series temporales de turbidez y clorofila- $a$ en el período 2002-2012 donde se observa un patrón singular, con aumentos abruptos de turbidez que desencadenan períodos con elevados valores de esta variable y baja concentración de clorofila- $a$ asociada. b) Espectro cruzado de series temporales de turbidez y clorofila-a de donde se concluye que estas dos variables tienen un período de oscilación común de 2,5 años pero con un desfasaje de unos 1,2 años (los ciclos de turbidez ocurren primero). c) Diagrama de dispersión de turbidez vs. clorofila- $a$. Se identifica un umbral de esta última variable por sobre el cual la clorofila-a presenta valores bajos. d) Árbol de clasificación para predicción de Grupos Funcionales Basados en Morfología en función de variables de calidad de agua y meteorológicas. Las distintas figuras fueron adaptadas de Crisci, et al. (en prensa).

la dinámica de procesos clave y generar modelos predictivos de biomasa y composición del fitoplancton.

\section{Sonda de registro continuo}

El sistema permite un seguimiento detallado del agua que ingresa a la planta de potabilización siendo capaz de generar alertas relevantes sobre cambios abruptos de calidad de agua en la laguna con una frecuencia mucho más elevada y un retardo mucho menor que cualquiera de las demás alternativas de monitoreo disponibles (Figura 4). No se han registrado floraciones fitoplanctónicas relevantes durante el período en el que la sonda ha estado instalada enviando datos.

Por estar instalada dentro de la planta, la sonda puede generar alarmas cuando el problema ya ingresó a ella (no hay anticipación, pero sí alerta temprana). En este sentido, resulta imprescindible la elaboración de protocolos de acción de mínimo plazo.

La interpretación de los datos y las alarmas generadas involucra una fase de exploración crítica y cruce de variables y la consideración de conocimiento teórico y experiencia previa sobre la dinámica del sistema. Por ejemplo, una alarma de muy elevado nivel de ficocianina, sin incremento de clorofila y turbidez, ni variaciones de oxígeno disuelto o $\mathrm{pH}$, tendría poco sentido y puede finalmente ser desestimada.

Para la interpretación de los cambios detectados en la calidad de agua resulta imprescindible tener acceso a información adicional a la generada por la sonda. La ausencia de esta información accesoria (metadatos) genera dificultades en la difusión de los datos generados, ya que pueden ser malinterpretados por carencia de información adicional.

Ejemplos:

- Cambios en el régimen de bombeo modifican las condiciones en el entorno de los sensores y deben ser tenidos en cuenta en el proceso de interpretación de los datos.

- Fenómenos meteorológicos modifican la calidad de agua. El acceso a tiempo real a la información meteorológica generada en la estación meteorológica permitiría descartar o asignar causas meteorológicas a cambios en la calidad de agua (por ejemplo, la disminución de conductividad asociada a lluvias copiosas, el aumento de evento de turbidez por viento, etcétera).

La Figura 4 presenta un ejemplo de salida gráfica de la sonda donde se observa la variación temporal de diversos parámetros de calidad de agua. 


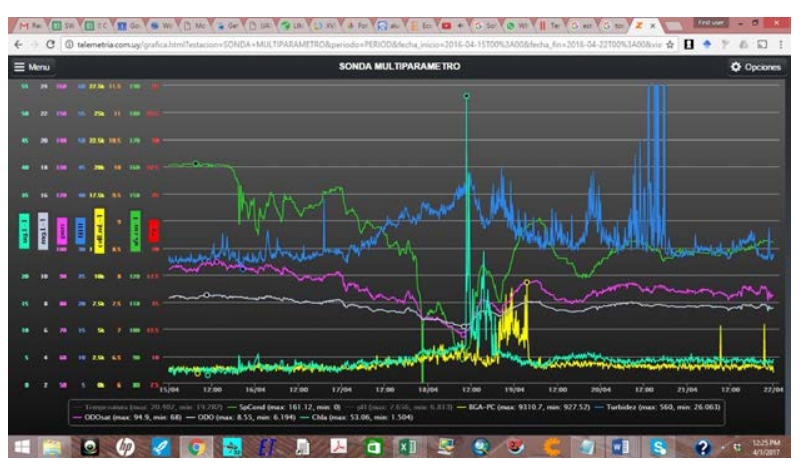

Figura 4. Ejemplo de salida gráfica de los datos de calidad de agua por parte de la sonda instalada en Laguna del Sauce. Se eligió el período entre el 15 y el 22/04/2016, ya que corresponde a una serie de eventos meteorológicos extremos. Se omitieron algunas variables para simplificar la visualización. Puede observarse la disminución abrupta de la conductividad asociada a lluvias copiosas (en verde), el incremento posterior de los valores de clorofila- $a$ (turquesa) y ficocianina en suspensión (amarillo) asociado al arrastre y resuspensión de perifiton, y el incremento de turbidez (azul) asociado a fuertes vientos.

\section{Muestreos semanales de información limnológica en el cuerpo de agua y red de tributarios}

Los muestreos semanales constituyen una respuesta reciente para poder comprender en mayor detalle la variabilidad espacial y temporal de las respuestas al proceso de eutrofización y en especial de las floraciones fitoplanctónicas. Llevan dos temporadas estivales en funcionamiento: 2015-2016 y 2016-2017. Los resultados dan cuenta de una considerable variabilidad interanual de floraciones en las épocas estivales (Figura 5). En la primera temporada se observó una sucesión de floraciones de cianobacterias potencialmente tóxicas que generaron cambios importantes en los niveles de oxígeno y $\mathrm{pH}$ durante las fases de crecimiento activo y colapso. En la segunda temporada pudo comprobarse la ocurrencia de floraciones de cianobacterias fijadoras de nitrógeno en Laguna del Potrero que no lograron expandirse al resto del sistema debido a las condiciones elevadas de turbidez. El diseño espacial y temporal del muestreo semanal ha resultado satisfactorio para comprender la dinámica del sistema y anticipar varios monitoreos adicionales (evaluación de toxinas) o incorporar tratamientos adicionales en el tratamiento de agua potable.

La Figura 5 da cuenta de la variación temporal de abundancia de cianobacterias en el subsistema Laguna del Sauce como resultado del monitoreo semanal.

\section{Muestreos mensuales de información$$
\text { limnológica en el cuerpo }
$$$$
\text { de agua y red de tributarios }
$$

El monitoreo mensual pretende comprender, con mayor resolución espacial que el muestreo semanal, las respuestas al proceso de eutrofización del sistema Laguna del Sauce. Se han

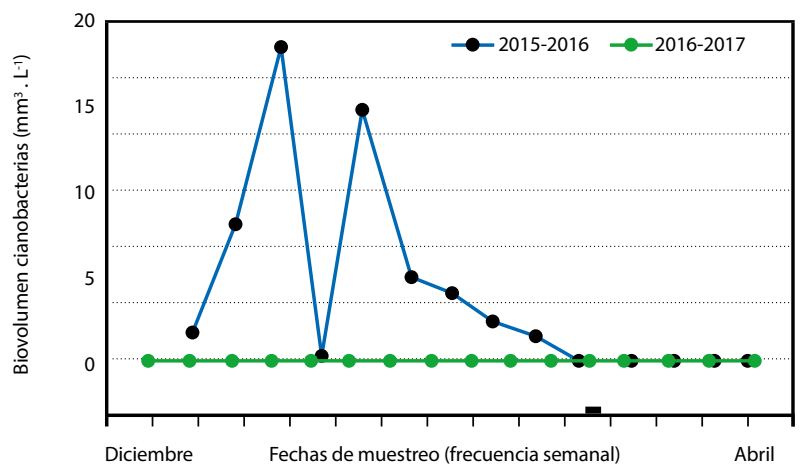

Figura 5. Variación temporal de la abundancia de cianobacterias (biovolumen de cianobacterias) en el subsistema Laguna del Sauce para los dos períodos considerados. En celeste: temporada diciembre 2015 - abril 2016; en verde: temporada diciembre 2016 - abril 2017.

podido comprender procesos clave en este sistema. El aporte de agua muy transparente del arroyo Pan de Azúcar, con niveles moderados a altos de nutrientes, favorece la dominancia de vegetación sumergida, componente que se encuentra en una fase de importante expansión espacial en los últimos 15 años. El tamaño de Laguna del Potrero y el fetch asociado limita la generación de grandes olas que dañen físicamente la vegetación acuática. Las condiciones en el sistema principal de Laguna del Sauce son sustancialmente diferentes debido a las diferencias de áreas, incidencia del viento y generación de olas. Este sistema carece de vegetación acuática, sus aguas presentan una turbidez inorgánica mayor y se encuentra dominado en algunos períodos por floraciones algales y de cianobacterias.

Los extensos humedales asociados al arroyo Pan de Azúcar y Sauce promueven los procesos de desnitrificación, gracias a lo cual se observan importantes fluctuaciones temporales en la concentración de nitrógeno total y muy bajas concentraciones en múltiples ocasiones. Estas condiciones favorecen el establecimiento de cianobacterias fijadoras de nitrógeno. En el caso particular de Laguna del Potrero, los bancos de plantas sumergidas, además de favorecer los procesos de desnitrificación, incorporan una parte significativa de los nutrientes por asimilación.

Este tipo de monitoreo ha permitido identificar tributarios con concentraciones altas de nutrientes en la cuenca, asociados a centros poblados con carencia de sistemas de saneamiento o emprendimientos productivos intensivos de tambos. Esta información es crucial para orientar espacialmente los esfuerzos en el control de las medidas recientemente adoptadas para manejar el aporte externo de nutrientes.

\section{Discusión}

El conjunto de las estrategias de monitoreo disponibles al día de hoy en Laguna del Sauce constituye un sistema de seguimiento clave para diversos procesos de análisis y toma de decisión. Como tal, responde a objetivos diversos, que se atienen a procesos que ocurren a distintas escalas espacio-temporales y, por tanto, la información se generará 
también a frecuencias y en escalas espaciales diferentes. La información de los monitores deriva en estrategias de prevención o mitigación de problemas de calidad de agua aplicables a distintas escalas (por ejemplo, agua bruta tomada por OSE-UGD, cuenca) pero también en la recopilación de información para el estudio de dinámicas clave del sistema y generación de modelos predictivos de calidad de agua que, a su vez, pretenden aportar a la mitigación y prevención, así como a la mejor interpretación de la información generada por diversas estrategias. Una de las características del sistema de seguimiento presentado es su carácter interinstitucional, lo que comprende un desafío desde diferentes puntos de vista. Asimismo, la necesidad de recursos humanos especializados para llevar adelante el monitoreo, registro y ordenación de la información y de recursos materiales para mantener en el tiempo ciertos aspectos del sistema de seguimiento constituye otro desafío relevante. En este contexto, se presentan algunos retos y perspectivas a futuro relacionados a las estrategias de monitoreo presentadas. A continuación se discute el interés de integrar nuevas estrategias de monitoreo a las ya existentes. Por último, se alude a las barreras a superar para mejorar el sistema de seguimiento actual.

\section{Registro de información hidrológica y meteorológica}

Resulta de interés estudiar la viabilidad de ampliar la cobertura pluviométrica en la cuenca de aporte para tener una mejor estimación del aporte a la laguna, tiempo de residencia del agua y evaluaciones adecuadas de las cargas externas de nutrientes.

Como se menciona previamente, a la fecha no existe una coordinación entre INUMET y OSE-UGD, de modo que los funcionarios de la usina cuenten con la información meteorológica (observada y de pronóstico) en tiempo real, ya sea de observación convencional como automática. Sería interesante poder coordinar mejor esta transferencia de información.

\section{Monitoreo de información limnológica en la planta de OSE-UGD}

Sería muy útil el almacenamiento de la información generada en un sistema informático único que esté a disposición de todos los generadores de información. Esto refiere a todas las estrategias de monitoreo (y las diferentes instituciones involucradas). Una posibilidad es que OSE-UGD lidere esta tarea. Esto conllevaría la contratación de recursos humanos adicionales porque se trata de una tarea demandante que difícilmente pueda ser llevada adelante por funcionarios que ya tienen responsabilidades asignadas.

\section{Sonda de registro continuo}

El mantenimiento del sistema de monitoreo+telemetría es una tarea exigente de recursos humanos e insumos (como, por ejemplo, repuestos y estándares), y su continuidad en el tiempo a mediano-largo plazo involucra la asignación de recursos adicionales.

La utilización de esta información en el proceso de toma de decisiones no es automática e implica una etapa imprescindible de interpretación y aprendizaje por parte de personal técnico especializado. A la fecha, esta capacidad no está asegurada.

\section{Muestreos semanales y mensuales de información limnológica en el cuerpo de agua y red de tributarios}

El esfuerzo de los muestreos semanales es considerable y por el momento se concentra en los períodos más críticos del año (verano). Este muestreo es posible en virtud de la cooperación y fondos aportados por la OSE-UGD y el CURE.

La posibilidad de poner en marcha este tipo de muestreo en otras épocas del año donde se registren floraciones fitoplanctónicas resulta inviable hoy, por la falta de un equipo destinado a esta tarea. Actualmente, tanto los muestreos semanales como mensuales son realizados por investigadores, estudiantes de posgrado y personal de OSE-UGD asignado a múltiples tareas. Cabe considerar la incorporación de recursos humanos permanentes al personal de OSE-UGD para desarrollar estos y otros tipos de monitoreo, colaborando al mismo tiempo con la actualización de las bases de datos y análisis de la información. En relación a los muestreos mensuales, la red de tributarios presenta dificultad de acceso, lo que limita la continuidad en el registro de la información por lo que es importante trabajar en sistemas de acceso a los campos y predios privados.

En los últimos años se adquirió una embarcación con fondos de OSE y del CURE para contar con medios destinados exclusivamente a estos muestreos. Esta estrategia ha resuelto varios inconvenientes en el pasado que generaron discontinuidades temporales en el monitoreo. Resulta conveniente pensar en un esquema similar para asegurar el acceso a la red de tributarios que requieren vehículos $4 \mathrm{X} 4$. Este recurso podría también utilizarse para las tareas de control y fiscalización.

\section{Otras alternativas de monitoreo a considerar}

En las últimas décadas se ha constatado un uso creciente de las geotecnologías aplicadas al estudio y evaluación de los recursos naturales y problemas ambientales. Las técnicas de sensoramiento remoto se han posicionado como una herramienta potente para el estudio de los sistemas acuáticos. En términos generales, este conjunto de técnicas permite conocer un objeto de estudio sin entrar en contacto con él, y generalmente refieren a la evaluación de objetos a partir de sensores localizados en plataformas espaciales (satélites) o aéreas (aeroplanos, helicópteros, drones, etcétera).

Entre las principales ventajas que brindan estas herramientas se destaca su eficiencia en costos económicos y tiempo, dado que brinda la posibilidad de realizar mediciones en períodos prolongados, con alta frecuencia y para amplias zonas, con costos menores a los relevamientos in situ. Además, permiten relevamientos en zonas de difícil acceso, y debido a su frecuencia y alcance espacial posibilitan identificar la variabilidad espacio-temporal de diversos fenómenos.

Si bien la utilidad y precisión de estas técnicas han sido ampliamente demostradas, su utilización no excluye la necesidad de aplicación de otros métodos, como los muestreos tradicionales. En este sentido, las técnicas de sensoramiento remoto para los estudios de calidad de agua, al igual que para el resto de las disciplinas científicas, son técnicas complementarias y no excluyentes.

Numerosos parámetros de calidad de agua pueden ser evaluados con estas técnicas. Dentro de los más estudiados 
se destacan clorofila- $a$, turbidez, temperatura, materia orgánica disuelta, sólidos en suspensión, salinidad, fósforo total, ortofosfatos, demanda química y demanda bioquímica de oxígeno, entre otros (Gholizadeh, et al., 2016).

En Uruguay son escasos los antecedentes de evaluaciones de parámetros de calidad de agua mediante técnicas de teledetección. La Comisión Administradora del Río Uruguay (CARU) (Uruguay-Argentina) ha realizado evaluaciones de clorofila- $a$ y temperatura del agua mediante la utilización de imágenes satelitales SPOT 5, LANDSAT 7TM y LANDSAT 8 OLI/TIRS en forma sistemática desde 2014 (Drozd, 2014-2016).

El sensoramiento remoto ha demostrado en nuestro país una gran utilidad para analizar la influencia de diversas características de cuencas de drenaje (tipo y uso de suelo, geología, geomorfología, vegetación riparia, entre otros) en la variación espacial de los niveles de nutrientes (Díaz, 2013) $\mathrm{u}$ otros atributos de la calidad del agua. Es importante desarrollar el componente de teledetección en el Sistema Laguna del Sauce y cuenca asociada, lo que permitirá contar con un seguimiento detallado de las principales transformaciones del uso del suelo y su incidencia en la calidad del agua de la red de tributarios. Al mismo tiempo, posibilitará analizar la trayectoria histórica (al menos desde la década de 1980 a la actualidad) de varios atributos clave de calidad del agua, por ejemplo, la recurrencia de las floraciones fitoplanctónicas, su origen en el espacio y su distribución espacial, expansión de la vegetación sumergida, entre muchos otros.

\section{Superando la fragmentación y promoviendo el aprendizaje}

Actualmente navegamos una transición múltiple en la gestión de los recursos acuáticos continentales asociada a cambios en el sistema de gobernanza, las considerables transformaciones del suelo y las múltiples crisis de calidad del agua en el suministro de agua potable (Mazzeo, et al., 2014). La dinámica temporal del fitoplancton (particularmente su variación en escalas de días y semanas) y la multiplicidad de factores que la gobiernan, por citar un ejemplo, plantean grandes desafíos al ámbito de la gestión, tanto en la detección de señales de alerta temprana como en la comprensión de las dinámicas de cambio. Para ello resulta necesario diseñar y articular múltiples programas de monitoreo con objetivos y escalas temporales y espaciales diferentes, en muchas ocasiones bajo la responsabilidad de instituciones y actores diferentes. La fragmentación en la generación de la información, así como los procesos de análisis y toma de decisión es, sin dudas, la primera gran barrera a superar. Posteriormente, se deben generar las condiciones que promueven el aprendizaje continuo, tanto de los fenómenos que afectan la calidad del agua como de los posibles tratamientos o recomendaciones de uso. Ese trayecto solo puede ser recorrido sobre la base de la confianza, la cooperación interinstitucional y la asignación de recursos originalmente no contemplados en el diseño institucional y de funciones.

\section{Reconocimientos}

Los autores agradecen a la Unidad de Gestión Desconcentrada de OSE en Laguna del Sauce (OSE-UGD), al Instituto Uruguayo de Meteorología (INUMET) y a la Dirección Nacional de Agua (DINAGUA) por el aporte de información y la buena disposición para el intercambio de ideas.

\section{Referencias}

Breiman, L., Friedman, J. H., Olshen, R. y Stone, C.J., 1984. Classification and regression trees. Monterey: Wadsworth

Breiman, L., 2001. Random forests. En: Machine Learning, 45(1), pp.5-32.

Carmichael, W. W., 2001. Health effects of toxin-producing cyanobacteria: «The CyanoHABs». En: Human and ecological risk assessment: An International Journal, 7(5), pp.1393-1407.

Carpenter, S. R., Stanley, E. H. y Vander Zanden, M. J., 2011. State of the world's freshwater ecosystems: physical, chemical, and biological changes. En: Annual Review of

Environment and Resources, 36, pp.75-99.

Comisión Administradora del Río Uruguay, 2015. Observación sistemática de la calidad del agua a lo largo del río Uruguay, (frontera argentino-uruguaya) mediante imágenes satelitales [En línea]. [s.l.]: CARU. (Informe 77). [Consulta 15 de marzo de 2017]. Disponible en: http://www.caru. org.uy/web/conae/Informe77.pdf

Clifton, H. E. y Dingler, J. R., 1984. Wave-formed structures and paleoenvironmental reconstruction. En: Marine Geology, 60(1-4), pp.165-198.

Crisci, C., Terra, R., Pacheco, J.P., Ghattas, B., Bidegain, M., Goyenola, G., Lagomarsino, J.J., Méndez, G. y Mazzeo, M., en prensa. Multi-model approach to predict phytoplankton biomass and composition dynamics in a eutrophic shallow lake. En: Ecological Modelling.

Díaz, I., 2013. Modelación de los aportes de nitrógeno y fósforo en cuencas hidrográficas del departamento de Canelones (Uruguay). Montevideo: Facultad de Ciencias. (Tesis de Maestría)

Elser, J. y Bennett, E., 2011. Phosphorus cycle: A broken biogeochemical cycle. En: Nature, 478, pp.29-31.

El Observador, 2015. Laguna del Sauce presenta un grado de contaminación elevada, según ministra [En línea]. En: $E l$ Observador. [Consulta 16 de abril de 2015]. Disponible en: http://www.elobservador.com.uy/laguna-del-saucepresenta-un-grado-contaminacion-elevada-segunministra-n302859.

Gholizadeh, M., Melesse, A. y Reddi, L., 2016. A comprehensive review on water quality parameters estimation using remote sensing techniques. En: Journal Sensors, 16(8), pp.1298-1306.

Gruber, N. y Galloway, J.N., 2008. An Earth-system perspective of the global nitrogen cycle. En: Nature, 451, pp.293-296.

Heisler, J., Glibert, P. M., Burkholder, J. M., Anderson, D. M., Cochlan, W., Dennison, Dortch, Q., Gobler, C. J., Heil, C. A., Humphries, E., Lewitus, A., Magnien, R., Marshall, H. G., Sellner, K., Stockwell, D.A., Stockwell, D. K. y Suddleson, M., 2008. Eutrophication and harmful algal blooms: a scientific consensus. En: Harmful algae, 8(1), pp.3-13.

Inda, H. y Steffen, M., eds., 2010. Bases técnicas para el manejo integrado de Laguna del Sauce y cuenca asociada. Montevideo: Editorial Montevideo. ISBN: 978-9974-00694-2.

Koroleff, F.,1970. Direct determination of ammonia in natural waters as indophenol blue. Information on techniques and methods for seawater analysis. Copenhagen: ICES. (Interlaboratory Report 3).

Kruk, C., Huszar, V. L., Peeters, E. T., Bonilla, S., Costa, L., Lürling, M., Reynolds, C. S. y Scheffer, M., 2010. A 
morphological classification capturing functional variation in phytoplankton. En: Freshwater Biology, 55(3), pp.614-627. Mazzeo, N., Steffen, M., Inda, H., Goyenola, G., Clemente, J., Teixeira de Mello, F., Iglesias, C., Pacheco, J.P. y Sciandro., J., 2014. Navegando una transición múltiple. En: Recursos hídricos: usos, tecnologías y participación social: un enfoque interdisciplinario. Montevideo: Espacio Interdisciplinario, Udelar. (Café y Tertulia; 2/13). ISBN 978-9974-0-1070-3.

Müller, R. y Widemann, O., 1955. Bestimmung des nitrations in Wasser. En: Vom Wasser, 22, pp.247.

Muñoz. A., 2015. La clave es el manejo [En línea]. En: La Diaria. [Consulta 16 de Junio de 2015]. Disponible en: https:// ladiaria.com.uy/articulo/2015/6/la-clave-es-el-manejo/.

Murphy, J. y Riley, J. P., 1962. A modified single solution method for the determination of phosphate in natural waters. En: Analytica Chimica Acta, 27, pp.31-36.
Nusch, E. A., 1980. Comparison of methods for chlorophyll and phaeopigment determination. En: Arch. Hydrobiol. Beih., Ergebn. Limnol, 14, pp.14-36.

Scheffer, M. y van Nes, E. H., 2007. Shallow lakes theory revisited: various alternative regimes driven by climate, nutrients, depth and lake size. En: Hydrobiologia, 584(1), pp.455-466.

Scheffer, M., Hosper, S. H., Meijer, M. L., Moss, B. y Jeppesen, E., 1993. Alternative equilibria in shallow lakes. En: Trends in Ecology \& Evolution, 8(8), pp.275-279.

Smith, V. H. y Schindler, D. W., 2009. Eutrophication science: where do we go from here? En: Trends in Ecology \& Evolution, 24(4), pp.201-207.

Valderrama, J. C., 1981. The simultaneous analysis of total nitrogen and total phosphorus in natural waters. En: Marine Chemistry, 10(2), pp.109-122. 